\title{
Repolarisation and refractoriness during early ischaemia in humans
}

\author{
P M I Sutton, P Taggart, T Opthof, R Coronel, R Trimlett, W Pugsley, P Kallis
}

\begin{abstract}
Objectives-To determine whether effective refractory period (ERP) shortens or lengthens in the first minutes of ischaemia in humans, and the relation between ERP changes and action potential duration (APD).

Methods-ERP and monophasic action potential duration (MAPD) were measured from a single left ventricular epicardial site in 26 patients undergoing coronary artery surgery. Cardiopulmonary bypass was instituted and normothermia maintained. Refractory period was determined by the extrastimulus technique at a basic cycle length of $500 \mathrm{~ms}$, at four times (group 1, 15 patients) or two times (group 2, 11 patients) the preischaemic diastolic threshold. A three minute period of ischaemia was instituted by aortic cross clamping between the input from the pump oxygenator and the heart.

Results-After three minutes of ischaemia, mean (SEM) ERP lengthened from 232 (5) ms (control) to $246(7) \mathrm{ms}(\mathrm{p}<0.005)$ in group 1 , and from $256(10) \mathrm{ms}$ (control) to 348 (25) $\mathrm{ms}$ $(\mathrm{p}<0.005)$ in group 2. In the same time MAPD shortened from 256 (5) ms (control) to 189 (9) $\mathrm{ms}(\mathrm{p}<0.001)$ with no difference between groups. Thus postrepolarisation refractoriness developed during ischaemia. Before ischaemia, ERP showed a good correlation with APD $\left(R^{2}=0.64\right)$ but by one minute of ischaemia the correlation was poor $\left(R^{2}=0.29\right)$.

Conclusions-These results show that during the first three minutes of global ischaemia in patients with coronary artery disease: (1) ERP lengthened in response to both a low and a high stimulus strength; and (2) there was a good correlation between ERP and APD before ischaemia, which was lost by one minute as APD decreased and ERP increased. These findings may have important implications in arrhythmogenesis.
\end{abstract}

(Heart 2000;84:365-369)

Keywords: refractoriness; ischaemia; repolarisation

Extensive experimental evidence implicates enhanced dispersion of refractoriness with arrhythmogenesis. ${ }^{12}$ A short refractory period interposed between regions of longer refractory period facilitates the formation of re-entrant circuits. ${ }^{3}$ Under normal conditions the refractory period is predominantly voltage dependent and ends at full repolarisation of the action potential. ${ }^{4}$ On this basis a common antiarrhythmic strategy is the use of drugs which prolong action potential duration (APD), with the intention of prolonging refractoriness and so reducing the likelihood of re-entry. ${ }^{5} \mathrm{APD}$ is widely used as a measure of refractoriness in studies related to mechanisms of arrhythmogenesis. However, experimental studies have shown that after the first few minutes of ischaemia the refractory period changes from being mainly voltage dependent to being mainly time dependent - that is, independent of APD..$^{6-11}$ This transition is relevant clinically, as the majority of serious ventricular arrhythmias during ischaemia are caused by re-entry and occur within the first minutes of onset. ${ }^{3}$ Information in humans is lacking. Extrapolation of experimental work on ischaemia induced changes in refractoriness is difficult because reports include a shortening, ${ }^{12-18}$ a lengthening, ${ }^{12}{ }^{19-21}$ a lengthening followed by a shortening, ${ }^{22}{ }^{23}$ or a shortening followed by a lengthening. ${ }^{9}{ }^{22}{ }^{24}{ }^{25}$ It is likely that at least some of these differences relate to methodological factors. These include the stimulus strength used in relation to the activation threshold, which increases progressively during early ischaemia, ${ }^{12}$ and the use of very strong stimuli which may excite fibres distant from the stimulation site. ${ }^{20}$ The presence or absence of residual perfusion (for example low flow ischaemia) or of diffusion across border zones between ischaemic and non-ischaemic regions may also be a factor. ${ }^{26}$ Extrapolation to the clinical situation is further hindered by the known electrophysiological species differences and a lack of comparative information between different animal models and patients with long standing coronary artery disease. The present study attempts to address some of these difficulties. We have performed studies directly in patients with chronic heart disease, used global ischaemia which eliminates border zones, and incorporated high and low pacing stimulus strengths. We sought answers to the following questions. First, in the initial few minutes of ischaemia in these patients, does the effective refractory period lengthen or shorten? Second, what is the relation of this change to $\mathrm{APD}$ ?

\section{Methods}

PATIENTS

We studied 26 patients (22 men and four women) aged 33-76 years (mean 63 years). All were undergoing routine coronary artery surgery for the relief of angina. Patients were selected at random. Patients taking cardioac- 
tive drugs other than $\beta$ blockers, calcium antagonists, and angiotensin converting enzyme (ACE) inhibitors were excluded. The study protocol was approved by the hospital ethics committee, and written informed consent was obtained from each patient.

EPICARDIAL MAP RECORDINGS

The monophasic action potential (MAP) provides a faithful representation of the time course of the transmembrane potential, ${ }^{27-29}$ and is an established and widely used technique for investigating the in situ beating heart. Reliable recordings may be obtained on the human epicardium. ${ }^{30}{ }^{31}$ We recorded an MAP using a pressure contact electrode from the anterior left ventricular epicardium. The MAP signals were fed to a Gould isolated preamplifier (model 11-5407-58) (Gould Electronics, Ilford, Essex, UK) to provide patient isolation and then to a Gould Universal amplifier (model 13-4615-58; input impedance $10^{9} \mathrm{ohms}$ ) with an output of $2.5 \mathrm{~V}$ for $40 \mathrm{mV}$ input. MAP signals were filtered from dc to $100 \mathrm{MHz}$. Data were digitised at a sampling rate of $1 \mathrm{kHz}$ (CED 1401 plus, Cambridge Electronic Design, Cambridge, UK) and stored.

PACING PROTOCOL

Patients were studied in two groups. There was no difference between the groups with respect to age, drug treatment, or number of coronary arteries with significant disease. Steady state cathodal pacing ( $2 \mathrm{~ms}$ current pulses, cycle length of $500 \mathrm{~ms}$ ) was established using a UHS 20 stimulator (Biotronik, Oxfordshire, UK). The pacing electrode was positioned on the left ventricular epicardium immediately adjacent to the MAP recording site, the rib spreaders acting as the anode. Stimulus strength was set
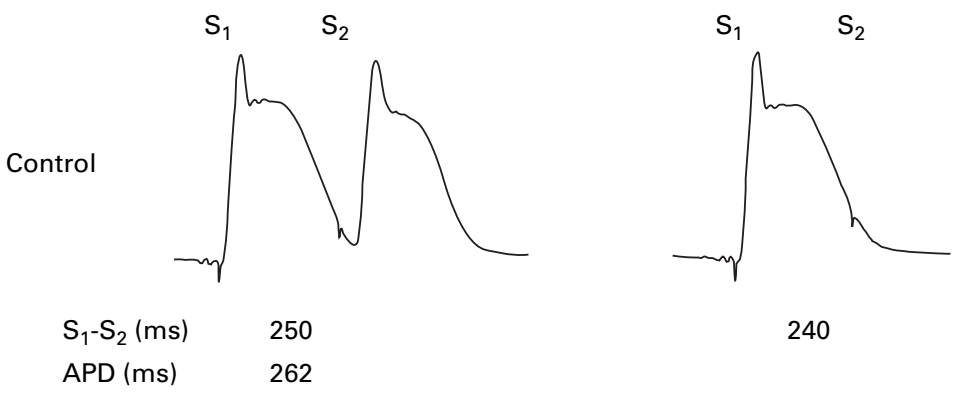

$\begin{array}{ll}\mathrm{S}_{1}-\mathrm{S}_{2}(\mathrm{~ms}) & 250 \\ \operatorname{APD}(\mathrm{ms}) & 262\end{array}$
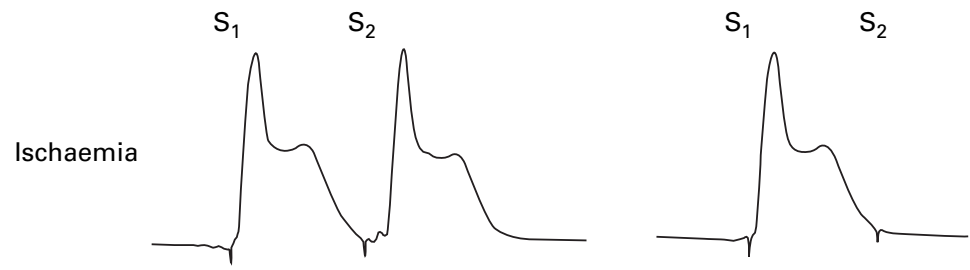

$\mathrm{S}_{1}-\mathrm{S}_{2}(\mathrm{~ms})$ 280

APD (ms)

230

Figure 1 Monophasic action potential (MAP) recordings (last $S 1$ and S2) during control (top) and ischaemia (below). The single MAPs on the right show the last MAP in the subsequent S1 train in which the S1-S2 interval is $10 \mathrm{~ms}$ shorter and S2 is unable to elicit a response. During ischaemia effective refractory period becomes longer than control and a gap is present between the repolarisation of the steady state beat (S1) and depolarisation of the premature beat (S2). at four times control diastolic threshold in one group of patients (group 1) and at two times control diastolic threshold in a second group of patients (group 2), and maintained at these (preischaemic) levels throughout the test period. Effective refractory period (ERP) was determined using the extrastimulus technique by decrementing a test pulse (S2) interval by $10 \mathrm{~ms}$ after each ninth basic beat (S1) until S2 did not result in an action potential or an ECG waveform. ERP was taken as the shortest possible S1-S2 interval that produced a propagated response.

\section{PROCEDURE}

Following the initiation of cardiopulmonary bypass and before revascularisation surgery, the electrodes were positioned, diastolic threshold determined, and electrophysiological measurements made. Normothermia was maintained. The cardiac surgeon routinely uses cross clamp fibrillation for the graft anastomoses. This involves clamping the ascending aorta between the inflow from the pump oxygenator and the heart. It has been shown that the resulting short period of ischaemia followed by reflow before the grafting induces a preconditioning effect and so is protective during the subsequent longer periods of ischaemia required for the graft procedures. ${ }^{32}$ We therefore used a three minute period of preconditioning ischaemia to measure the changes in refractory period and APD. After three minutes the cross clamp was released and pacing discontinued, following which routine surgery proceeded.

\section{ANALYSIS OF DATA}

MAP signals were measured using an interactive computer program, and measurements of APD were made at $90 \%$ repolarisation. This allowed a visual inspection of the computer measurements for each complex measured. Statistical evaluation used analysis of variance with Bonferroni correction for multiple comparisons (SPSS statistical package). Data are given as mean (SEM).

\section{Results}

Figure 1 shows MAP recordings illustrating several features of the effects of ischaemia on APD and ERP. Recordings before ischaemia are shown in the upper panel. The top left pair of MAP signals is the last of an S1 train followed by the test action potential at the shortest S1-S2 coupling interval $(250 \mathrm{~ms})$ which elicited a propagated action potential. The top right MAP is the last $\mathrm{S} 1$ action potential at a shorter S1-S2 interval $(240 \mathrm{~ms})$ which fails to elicit an action potential. The refractory period is therefore $250 \mathrm{~ms}$. The effect of ischaemia is shown in a similar format in the lower panel. The last S1 APD had shortened (from $262 \mathrm{~ms}$ to $230 \mathrm{~ms}$ ) while the shortest S1-S2 interval at which a propagated action potential could be achieved was now longer $(280 \mathrm{~ms})$. The "take off" of the test action potential following the S2 stimulus during control occurred before final repolarisation of the S1 action potential (top left). During 
ischaemia the test action potential upstroke occurred after full repolarisation owing to postrepolarisation refractoriness (bottom right).

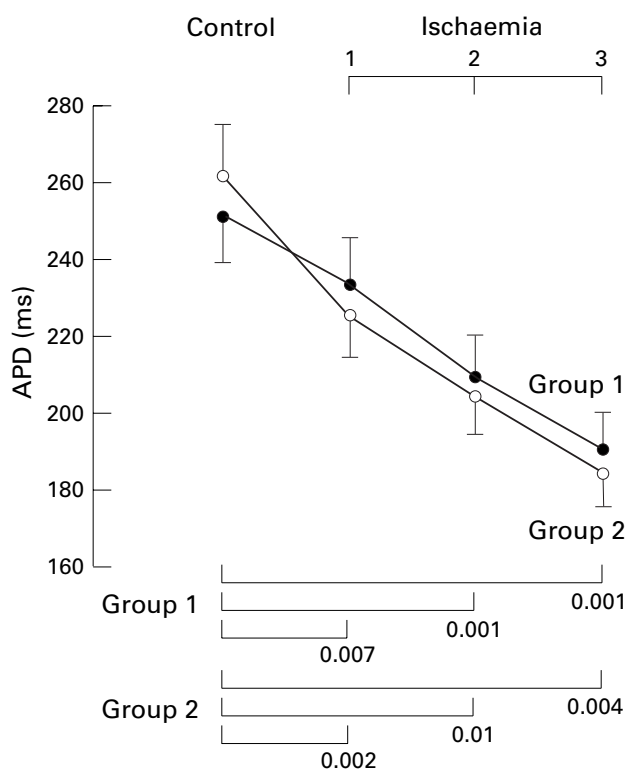

Figure 2 Monophasic action potential at 90\% repolarisation $\left(M A P D_{90}\right)$ of the left ventricular epicardium in patients with coronary artery disease during control conditions and after one, two, and three minutes of ischaemia (cycle length 500 ms). Values are shown separately for group 1, paced at four times preischaemic diastolic threshold, and group 2, paced at two times preischaemic diastolic threshold. Action potential duration (APD) shortened progressively during ischaemia. Values are means, error bars $=S E M$.

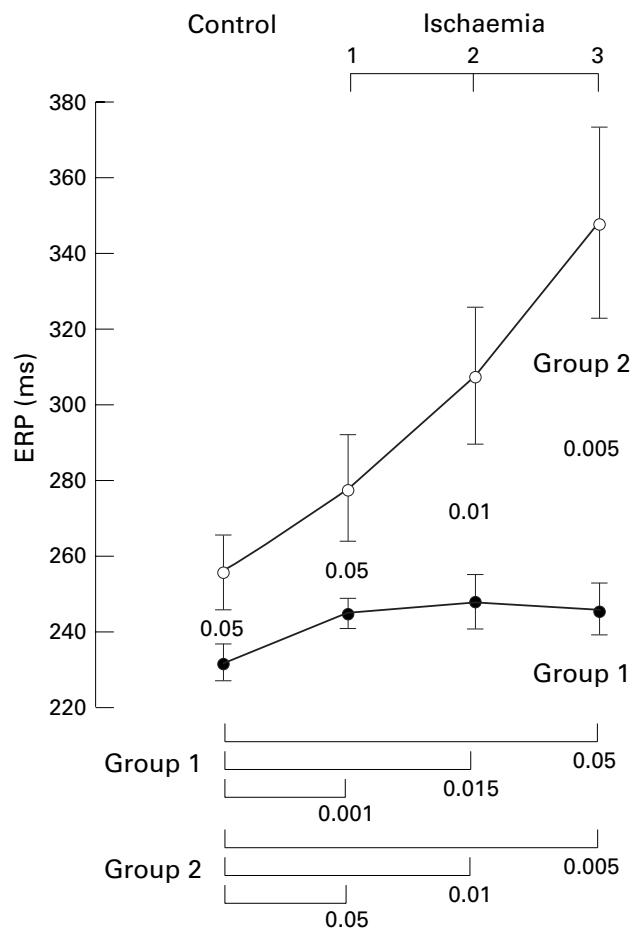

Figure 3 Effective refractory period (ERP) on the left ventricular epicardium in patients with coronary artery disease during control conditions and after one, two, and three minutes of ischaemia. Mean values for ERP showed a small increase during ischaemia in group 1 (high pacing stimulus) and a substantial increase in group 2 (low pacing stimulus). Values are means, error bars = SEM.
ACTION POTENTIAL DURATION

In all patients studied, MAP duration at $90 \%$ repolarisation $\left(\mathrm{MAPD}_{90}\right)$ shortened during ischaemia. Mean values for all patients were 256 (5) ms (control), 231 (6) ms ( $<<0.001$ ) at one minute, 209 (8) $\mathrm{ms}$ at two minutes $(\mathrm{p}<0.001)$, and $189(9) \mathrm{ms}(\mathrm{p}<0.001)$ at three minutes of ischaemia. Figure 2 shows that the values for group 1 patients (high stimulus strength) do not differ from those in group 2 patients (low stimulus strength).

REFRACTORY PERIODS

In contrast to the MAPD shortening, ERP increased at both stimulus strengths. Figure 3 shows mean values increasing from 232 (5) $\mathrm{ms}$ (control) to 245 (4) $\mathrm{ms}$ ( $\mathrm{p}<0.001)$, to 248 (7) $\mathrm{ms} \quad(\mathrm{p}<0.015)$, and to 246 (7) $\mathrm{ms}$ $(\mathrm{p}<0.05)$ in group 1 , and from 256 (10) ms (control) to $278(14) \mathrm{ms}(\mathrm{p}<0.05)$, to 308 (18) $\mathrm{ms} \quad(\mathrm{p}<0.01)$, and to 348 (25) $\mathrm{ms}$ $(\mathrm{p}<0.005)$ in group 2, after one, two, and three minutes of ischaemia, respectively. The increase in refractory period was significant from one minute of ischaemia onwards for both groups.

POSTREPOLARISATION REFRACTORINESS

Before ischaemia ERP was shorter than APD. Figure 4 (left) shows that the difference between ERP and APD was -20 (5) $\mathrm{ms}$ for group 1 and -8 (3) ms for group 2. During ischaemia this relation reversed, with ERP becoming longer than APD: 10 (7) ms (group 1) and 41 (9) $\mathrm{ms}$ (group 2) at one minute of ischaemia; 35 (7) ms (group 1) and 92 (14) ms (group 2) at two minutes; and 48 (9) $\mathrm{ms}$ (group 1) and 137 (19) ms (group 2) at three minutes $(p<0.001$ at all time points for both groups). The development of postrepolarisa-

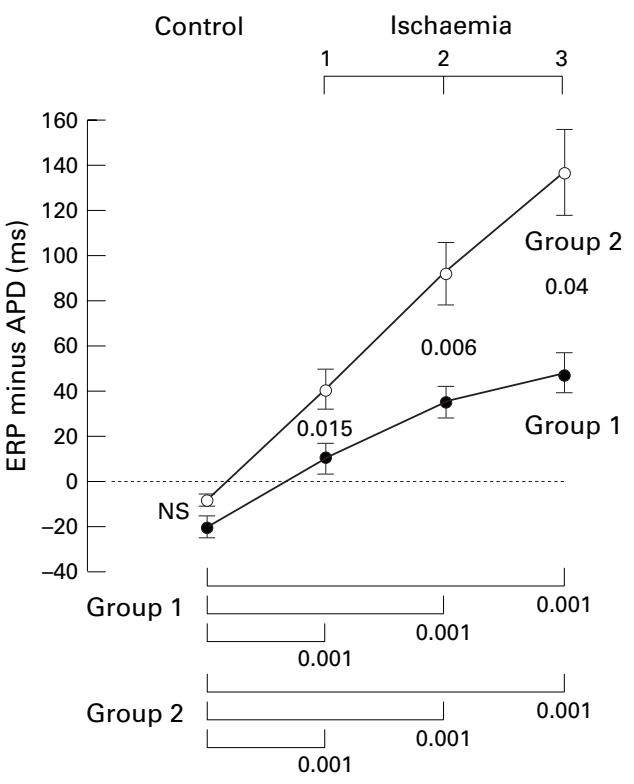

Figure 4 The relation between action potential duration (APD) and refractoriness is shown as effective refractory period (ERP) minus APD for group 1 and group 2

patients. Under control conditions ERP was shorter than APD. During ischaemia this relation rapidly reversed, ERP outlasting APD (postrepolarisation refractoriness). This effect is more pronounced in group 2 patients (low stimulus strength). 
tion refractoriness reflected the combined effect of APD shortening (fig 2) and ERP lengthening (fig 3). The latter was more pronounced in group 2 patients. During control the relation between ERP and APD showed a good correlation $\left(R^{2}=0.64\right.$, $\mathrm{p}<0.001)$, which was lost in the course of ischaemia $\left(R^{2}=0.29, \mathrm{p}<0.01 ; R^{2}=0.27\right.$, $\mathrm{p}<0.02$; and $R^{2}=0.13, \mathrm{p}<0.14$ at one, two, and three minutes, respectively).

\section{Discussion}

We found that ERP lengthened during a short period of global ischaemia in the human heart following pacing with high and low stimulus strength. The high stimulus strength resulted in less ERP prolongation. It is known that refractoriness depends on APD and becomes dissociated from it during ischaemia. ${ }^{6-11}$ We therefore investigated the relation between ERP and APD in the human heart. Under control conditions ERP approximated MAPD, and there was a good correlation between the two. During ischaemia MAPD shortened while ERP increased, resulting in postrepolarisation refractoriness from the first minute of ischaemia onward.

Previous studies on ischaemia induced changes reported lengthening ${ }^{12-21}$ or shortening ${ }^{12-18}$ of refractoriness. These differences may relate to methodological aspects of ERP measurement in ischaemia. Indeed, in dogs ERP either lengthened or shortened depending on the stimulus intensity. ${ }^{12}$ During ischaemia, stimulus threshold increases following an initial decrease $\mathrm{e}^{12} 2033$ depending on the site of stimulation relative to the ischaemic border. ${ }^{33}$ Thus most investigators use a fixed stimulus strength ranging from twice to 15 times diastolic threshold. ${ }^{15}$ However, in regional ischaemia high intensity stimulation may inadvertently excite distant myocardium where ERP is shorter and excitability is increased. ${ }^{20} 2133$ We therefore chose a model of global ischaemia, where border zone related phenomena are absent, and used two stimulus strengths. In humans ERP prolongation occurred over a wide range of stimulus strength.

Postrepolarisation refractoriness occurs in various models of ischaemia, ${ }^{6-11}$ although it has hitherto not been described in humans. It is caused by the combined effects of hypoxia and the accumulation of extracellular potassium $\left(\mathrm{K}_{\mathrm{o}}\right) .{ }^{113435}$ In border zone tissue where the rise in $K_{0}$ is moderate, ${ }^{36}$ refractory periods shorten. ${ }^{20} 2137$ In the centre of the ischaemic area $K_{0}$ is similar to that found in global ischaemia. ${ }^{26}$ The resting membrane potential ${ }^{38}$ and the recovery of excitability ${ }^{7}$ show a corresponding gradient from the periphery to the centre. Thus we speculate that the factors that influence $\mathrm{K}_{\mathrm{o}}$, such as diffusion and residual perfusion during low flow ischaemia, ${ }^{39}$ would influence ERP and the relation of ERP to APD. This may result in local ERP differences.

The observed changes in MAPD and refractoriness in response to ischaemia show a much faster time course in patients (with ischaemic heart disease) than in experimental animals. ${ }^{40}$ This highlights the importance of performing these studies directly in patients. Some aspects of methodology require mention. Our observations on MAPD/ERP were confined to a single left ventricular site in each patient. MAPD varies at different ventricular sites ${ }^{41}{ }^{42}$ and the effects of ischaemia are heterogeneous ${ }^{26}$ as well. However, our study employed global ischaemia which substantially reduces heterogeneity, and the recording sites were selected randomly on the left ventricular anterior wall, with consistent findings within each group. Also, the patients were on cardiopulmonary bypass and therefore the hearts were in a non-working state. This would have reduced the ischaemic burden and the influence of mechanoelectric feedback. $^{31}$ As anticipated from the small heterogeneity, no arrhythmias occurred during ischaemia in these hearts.

This study examines one end of a spectrum of ischaemia-that is, zero perfusion/zero diffusion-in contrast to border zones of regional ischaemia or low flow ischaemia. Although extrapolation to the clinical setting requires caution, we would suggest that the results represent the central ischaemic zone where perfusion is absent and diffusion gradients are small. ${ }^{26}$

Mechanisms of arrhythmia are frequently interpreted and modelled using APD as a measure of ERP. Our results suggest that while the relation between APD and ERP may persist during early ischaemia in peripheral regions, ERP would be prolonged beyond repolarisation in central areas. These findings may be relevant to class III antiarrhythmic agents $^{34} 35434$ and APD alternans as precursor of ventricular fibrillation. ${ }^{45}{ }^{46}$ Also, recent studies have centred on the role of the cycle length dependence of APD in the breakup of spiral waves into multiple wavelets characteristic of ventricular fibrillation. ${ }^{45-48}$ This has the potential for an alternative antiarrhythmic strategy, namely altering the slope of the APD/ERP relation. ${ }^{47}{ }^{48}$ The early dissociation of APD and ERP within the first minutes of ischaemia, at a time when re-entrant arrhythmias develop, would be expected to alter the dynamics of the relation. Furthermore, our results and the above discussion suggest that this effect would be inhomogeneous.

This work was supported by the British Heart Foundation (PT).

1 Han J, Moe GK. Non-uniform recovery of excitability of ventricular muscle. Circ Res 1964;14:44-60.

2 Kuo CS, Manakata K, Reddy CP, et al. Characteristics and possible mechanism of ventricular arrhythmia dependent on the dispersion of action potential duration. Circulation 1983;67:1356-67.

3 Janse MJ, Wit AL. Electrophysiological mechanisms of venricular arrhythmias resulting from myocardial ischaemia and infarction. Physiol Rev 1989;69:1049-169.

4 Hoffman BF, Cranefield PF. Electrophysiology of the heart. New York: McGraw-Hill, 1960.

5 Task Force of the Working Group on Arrhythmias of the European Society of Cardiology. The Sicilian Gambit. A new approach to the classification of antiarrhythmic drugs based on their actions on arrhythmogenic mechanisms. Circulation 1991;84:1831-51.

6 Cranefield P, Wit AL, Hoffman BF. Conduction of the cardiac impulse. Characteristics of very slow conduction. $f$ Gen Physiol 1972;59:227-46.

7 Gettes LS, Reuter H. Slow recovery from inactivation of inward currents in mammalian myocardial fibres. $\mathcal{F}$ Physiol (Lond) 1974;240:703-24.

8 El Sherif N, Scherlag BJ, Lazzara R, et al. Pathophysiology of El Sherif N, Scherlag BJ, Lazzara R, et al. Pathophysiology of
tachycardia and bradycardia dependent block in the canine 
proximal His-Purkinje system after acute myocardial ischemia. Am f Cardiol 1974;33:529-40.

9 Downar E, Janse MJ, Durrer D. The effect of acute coronary artery occlusion on subepicardial transmembrane potentials in the intact porcine heart. Circulation 1977;56:21724.

10 Lazzara R, El-Sherif N, Hope RR, et al. Ventricular arrhythmias and electrophysiological consequences of myocardial ischemia and infarction. Circ Res 1978;42:740-9.

11 Kodama I, Wilde AAM, Janse MJ, et al. Combined effects of hypoxia, hyperkalemia and acidosis on membrane action potential and excitability of guinea pig ventricular muscle. f Mol Cell Cardiol 1984;16:246-59.

12 Elharrar V, Foster PR, Jirak TL, et al. Alterations in canine myocardial excitability during ischemia. Circ Res 1977;40: 98-105.

13 Kuppersmith J, Antman EM, Hoffman BF. In vivo electrophysiological effects of lidocaine in canine acute myocarphysiological effects of lidocaine in can

14 Ramanathan KB, Bodenheimer M, Banka VS, et al. Electrophysiological effects of partial coronary occlusions and physiological effects of partial coronary
reperfusion. Am $\mathcal{F}$ Cardiol 1977;40:50-4.

15 Batsford WPD, Cannon DS, Zaret BI. Relationship between ventricular refractoriness and regional myocardial blood flow after acute coronary occlusion. Am 7 Cardiol 1978;41 1083-8.

16 Stewart JR, Burmeister WE, Burmeister J, et al. Electrophysiologic and antiarrhythmic effects of phentolamine in experimental coronary artery occlusion and reperfusion in the dog. F Cardiovasc Pharmacol 1980;2:77-81.

17 Kimura S, Bassett AL, Kohya T, et al. Simultaneous recording of action potentials from endocardium and epicardium during ischemia in the isolated cat ventricle: relation of temporal; electrophysiologic heterogeneities to arrhythmias. Circulation 1986;74:401-9.

18 Wolk R, Cobbe SM, Hicks MN, et al. Effects of lignocaine on dispersion of repolarisation and refractoriness in a working rabbit heart model of regional myocardial ischaeworking rabbit heart model of regional myocard

19 Horacek T, Neumann M, Van Mutius S, et al. Nonhomogeneous electrophysiological changes and the bimodal distribution of early ventricular arrhythmias during acute coronbution of early ventricular arrhythmias during acute
ary occlusion. Basic Res Cardiol 1984;79:649-67.

20 Janse MJ, Capucci A, Coronel R, et al. Variability of recovery in the normal canine and the ischaemic porcine heart. Eur Heart f 1985;6(suppl D): 41-52.

21 Capucci A, Coronel R, Fabius M, et al. Electrophysiologic mechanisms of ventricular arrhythmias in acute ischemia. New Trends Arrhythmias 1995;1:41-56.

22 Russell DG, Oliver MF. Ventricular refractoriness during acute myocardial ischaemia and its relationship to ventricular fibrillation. Cardiovasc Res 1978;12:221-7.

23 Penny WJ. The deleterious effects of myocardial catecholamines on cellular electrophysiology and arrhythmias dur73 .

24 Han J, Goel BG, Hanson CS. Reentrant beats induced in the ventricle during coronary occlusion. Am Heart f 1980; 80:778-84.

25 Gilmour RF, Zipes DP. Different electrophysiological responses of canine endocardium and epicardium to combined hyperkalemia, hypoxia and acidosis. Circ Res 1980;46:814-25.

26 Coronel R, Fiolet JWT, Wilms-Schopman FJG, et al. Distribution of extracellular potassium and its relation to electrophysiologic changes during acute myocardial ischemia in the isolated perfused porcine heart. Circulation 1988;77: $1125-38$

27 Hoffman BF, Cranefield PF, Lepeschkin E, et al. Comparison of cardiac monophasic action potentials recorded by intracellular and suction electrodes. Am f Physiol 1959;196: 1297-301.

28 Franz MR, Burkhoff D, Spurgeon $\mathrm{H}$, et al. In vitro validation of a new cardiac catheter technique for recording
monophasic action potentials. Eur Heart f $1986 ; 7: 34-41$.
29 Ino $\mathrm{T}$, Karagueuzian HS, Hong $\mathrm{K}$, et al. Relation of monophasic action potentials recorded with contact electrode to underlying transmembrane action potential properties in isolated cardiac tissues: a systematic microelectrode validation study. Cardiovasc Res 1988;22:255-64.

30 Taggart P, Sutton P, Runnalls M, et al. Use of monophasic action potential recordings during routine coronary artery bypass surgery as an index of localised myocardial ischaemia. Lancet 1986; i:1462-4.

31 Taggart P, Sutton PMI, Treasure T, et al. Monophasic action potentials at discontinuation of cardiopulmonary bypass: evidence for contraction-excitation feedback in man. Circulation 1988;77:1266-75.

32 Yellon DM, Alkulaifi AM, Pugsley WB. Preconditioning the human myocardium. Lancet 1993;342:276-7.

33 Coronel R, Wilms-Schopman FJG, Opthof T, et al. Injury current and gradients of diastolic stimulation threshold, TQ potential, and extracellular potassium concentration during acute regional ischemia in the isolated perfused pig heart. Circ Res 1991;68:1241-9.

34 Cobbe SM, Manley BS. Effects of elevated extracellular potassium concentrations on the class III antiarrhythmic action of sotalol. Cardiovasc Res 1985;19:69-75.

35 Cobbe SM, Manley BS, Alexopoulos D. The influence of acute myocardial ischaemia on the class III antiarrhythmic action of sotalol. Cardiovasc Res 1985;19:661-7.

36 Fleet WF, Johnson TA, Graebner CA, et al. Effect of serial brief ischemic episodes on the extracellular $\mathrm{K}^{+}, \mathrm{pH}$, and activation in the pig. Circulation 1986;72:922-32.

37 Janse MJ, Cinca J, Morena H, et al. The "border zone" in myocardial ischemia. An electrophysiological, metabolic and histochemical correlation in the pig heart. Circ Res 1978;44:576-88

38 Kléber A. Resting membrane potential, extracellular potassium activity and intracellular sodium activity during acute global ischemia in isolated perfused guinea pig hearts. Circ Res 1983;52:442-50.

39 Wantanabe I, Johnson TA, Buchanan J, et al. Effect of graded coronary flow reduction on ionic, electrical and mechanical indices of ischemia in the pig. Circulation 1987; 76:1127-34

40 Coronel R, Opthof $\mathrm{T}$, Taggart $\mathrm{P}$, et al. Differential electrophysiology of repolarisation from clone to clinic. Cardiovasc Res 1997;33:503-17.

41 Campbell-Cowan J, Hilton CJ, Griffiths CJ, et al. Sequence of epicardial repolarisation and configuration of the $\mathrm{T}$ wave. Br Heart f 1988;60:424-33.

42 Franz MR, Bargheer K, Rafflenbeul W, et al. Monophasic action potential mapping in human subjects with normal electrocardiograms: direct evidence for the genesis of the $\mathrm{T}$ wave. Circulation 1987;75:379-86.

43 Cobbe SM, Manley BS. The influence of ischaemia on the electrophysiological properties of amiodarone in chronically treated rabbit hearts. Eur Heart $\mathcal{F} 1987 ; 8: 1241-8$.

44 Culling W, Penny WJ, Sheridan DJ. Effects of sotalol on arrhythmias and electrophysiology during myocardial ischaemia and reperfusion. Cardiovasc Res 1984;18:397404 .

45 Koller ML, Riccio ML, Gilmour RF. Dynamic restitution of action potential duration during electrical alternans and ventricular fibrillation. Am f Physiol 1999;275:H1635-42.

46 Pastore JM, Giroward SD, Laurita KR, et al. Mechanism linking T-wave alternans to the genesis of cardiac fibrillation. Circulation 1999;99:1385-94.

47 Gilmour RF, Chialvo DR. Electrical restitution, critical mass and the riddle of fibrillation. $\mathcal{F}$ Cardiovasc Electrophysiol 1999;10:1087-9.

48 Qu Z, Weiss JN, Garfinkel A. Cardiac electrical restitution properties and stability of reentrant spiral waves: a simulation study. Am f Physiol 1999;276:H269-83. 